LA W REN CE LIVERM ORE N A TIONAL LABORATORY
Size-dependent Si Nanowire Mechanics are Invariant to Changes in the Surface State

B. Lee, R. E. Rudd

January 4, 2011

Nature Materials 
This document was prepared as an account of work sponsored by an agency of the United States government. Neither the United States government nor Lawrence Livermore National Security, LLC, nor any of their employees makes any warranty, expressed or implied, or assumes any legal liability or responsibility for the accuracy, completeness, or usefulness of any information, apparatus, product, or process disclosed, or represents that its use would not infringe privately owned rights. Reference herein to any specific commercial product, process, or service by trade name, trademark, manufacturer, or otherwise does not necessarily constitute or imply its endorsement, recommendation, or favoring by the United States government or Lawrence Livermore National Security, LLC. The views and opinions of authors expressed herein do not necessarily state or reflect those of the United States government or Lawrence Livermore National Security, LLC, and shall not be used for advertising or product endorsement purposes. 


\title{
Size-dependent Si nanowire mechanics are invariant to changes in the surface state
}

\author{
Byeongchan Lee \\ Kyung Hee University, Yongin, Gyeonggi 446-701, Republic of Korea \\ Robert E. Rudd* \\ Lawrence Livermore National Laboratory, L-045, Livermore, California 94551, USA
}

(Dated: September 18, 2011)

\begin{abstract}
We investigate the mechanics of $\mathrm{Si}$ nanowires using first-principles theory and find that the nanowires exhibit the same softening (decreased Young's modulus) as the wire diameter is reduced regardless of the surface reconstruction or passivation. This invariance is contrary to the expectation that the lower coordination of the bare surfaces affects the bond order and leads to stiffer nanowires than the H-passivated nanowires. We rigorously connect electronic structures and mechanical properties to show why the Young's modulus is insensitive to the surface state.
\end{abstract}

PACS numbers: 68.35.Gy, 62.25.-g, 85.85.+j

Nanoscale systems behave differently than their macroscopic counterparts in many aspects. One of these is that mechanical properties are anomalous at the nanoscale, a fact important in the design and development of nano-electro-mechanical systems (NEMS). Semiconductor nanowires used as the active element in nanoscale mechanical resonators can act as extremely sensitive detectors,${ }^{1-3}$ provided the mechanical behavior of the devices is understood. The mere presence of surface affects many properties of Si nanowires: the ground-state shape changes ${ }^{4}$ and even the band gap disappears ${ }^{5}$ in spite of the inherent gap-opening due to quantum confinement. Potentially small changes to the surface state of the resonator could alter its performance in unpredictable ways. Here we show the mechanical properties of Si nanowires are systematically size-dependent, but in a way that is remarkably insensitive to the surface state.

Previously classical molecular dynamics (MD) simulation has been used to study the mechanics of nanowires, finding size-dependent properties linked to the behavior of the surface material. ${ }^{6,7}$ The size dependence of the Young's modulus has also been related to continuum properties such as surface stresses ${ }^{8}$ or surface elastic constants. ${ }^{9,10}$ It must ultimately be related to properties of the electronic bonding, and some attempts have been made to explain this in connection with a charge redistribution on the surface, ${ }^{11}$ and empirical bond order effects due to the reduced coordination of surface atoms. ${ }^{11,12}$ Classical MD simulations of $\mathrm{Si}$ nanowires show striking size-dependent mechanical effects, but the specific mechanism and even the sign of the effect depend on which interatomic potential is used. Tersoff potentials, with strong bond-order effects, predict an increase in the Young's modulus (stiffening) of $\mathrm{Si}<001>$ nanowires with bare reconstructed surfaces as the size is decreased, ${ }^{13}$ whereas the Stillinger-Weber potential results in softening. ${ }^{10}$ Simulations of metallic nanowires show a stiffening ${ }^{9,14}$ similar to the Tersoff prediction for $\mathrm{Si}$. Classical potentials are used widely, and yet it is an open question as to what extent they can model the relevant physics for the simulation of nanowire mechanics.
Here we study the mechanical properties of bare Si nanowires and compare to the properties of hydrogenpassivated Si nanowires, both calculated with Density Functional Theory (DFT), to understand the effect of the surface state on mechanical properties. ${ }^{10}$ High fidelity calculations up to relatively large system sizes go well beyond prior theoretical studies to determine the systematic variation of nanowire properties. We consider $<001>$ nanowires due to the relevance to NEMS. The core is taken to be crystalline as found in experiment. ${ }^{15}$ The surface is taken to be faceted according to the Wulff shape with two types of low energy facets: $\{100\}$ and $\{110\}$. Stable surface reconstructions are known for both facets, ${ }^{16-20}$ and the (100)-p(2x2) and (110)-(1x1) reconstructions have been chosen here. For the (110) facet, we also consider the (110)-(1x2) pattern in a few cases for comparison. This surface exhibits a band gap whereas the (110)-(1x1) surface is metallic. ${ }^{20}$ For the (100) facet a larger pattern, (100)-(4x2) with a missing dimer, is known to be slightly more stable than, but comparable in energy to, the (100)-p(2x2) reconstruction at zero temperature. At room temperature, however, such a large ordering may not be stable so that dimers would be the dominant pattern on the (100) surface. ${ }^{17}$ The fully relaxed structures and the energetics have been obtained ${ }^{21}$ with the Vienna ab-initio simulation package (VASP) ${ }^{22}$ using the projector augmented-wave method ${ }^{23,24}$ within the generalized gradient approximation. ${ }^{25}$

The longitudinal Young's moduli of the $\mathrm{Si}\langle 001\rangle$ nanowires with bare and hydrogen-passivated surfaces ${ }^{10}$ are compared in Fig. 1. It is striking that the two data sets completely overlap with the one exception of the bare 1.13-nm wire. In both cases the Young's modulus decreases as the wire width is reduced. As we discuss in detail below, other properties of the bare wires do not show this smooth systematic size dependence, let alone agreement with the H-passivated wire properties. Both the band gap and the surface stress (and resulting eigenstrain) vary considerably for the same wires showing the smooth trend in the Young's modulus. The insensitivity of the scaling curve to surface passivation and surface 


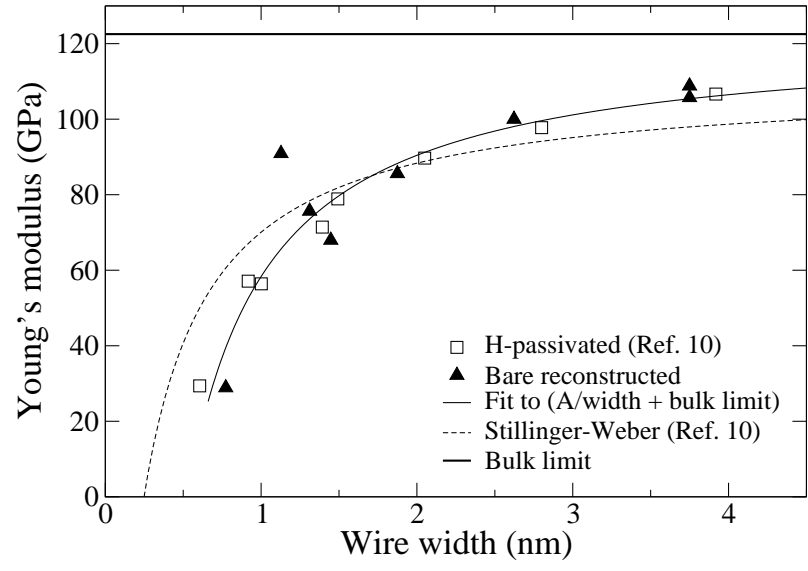

FIG. 1: Calculated Young's modulus of $\mathrm{Si}<001>$ nanowires as a function of wire width. The empirical values for bare wires and the first principles values for $\mathrm{H}$-passivated wires from our previous work (Ref. 10) are shown for comparison. The solid curve is a fit of all the first principles data except the outlying bare 1.13-nm wire. The width of a wire is defined as the square root of the cross-sectional area (Ref. 10).

stress is unexpected, and it suggests the absence of the effect of bond order on the Young's modulus. We are motivated then to consider the origin of the mechanical properties in the electronic structure of the wires, and to consider the character of the bonding and the role of bond order $(\mathrm{BO})$ to explain this unconventional result.

The nature of the bonding can be investigated through electron localization functions (ELFs) and bond order. ELFs provide a means of chemical bond classifications by a topological analysis based on the Pauli exclusion principle. ${ }^{26,27}$ The ELF analysis in Fig. 2(a)-(b) clearly shows two aspects of bonding. First, a bonding attractor, appearing as spherical isosurfaces between pairs of neighboring atoms, characterizes the localized shared-electron interaction of the covalent bond. Second, a non-bonding attractor, appearing as balloon-like isosurfaces at the surface atoms, is formed by the less but still localized electrons from a lone pair at the surface. The electrons from a dangling bond, without noticeable charge redistribution to neighboring bonds, remain where the missing bond would have been. This is opposite to the case of metallic surfaces in which charge redistribution induces a BO increase for surface bonds, and hence, surface stiffening. ${ }^{11}$

In the absence of significant charge redistribution on the Si nanowire surface, the bond order change at the surface is not pronounced. Bond order is obtained from the solution of the Schrödinger equation

$$
\hat{H}\left|\psi_{n}\right\rangle=\epsilon_{n}\left|\psi_{n}\right\rangle
$$

where $\left|\psi_{n}\right\rangle$ is an eigenstate ( $n$-th band) of the Hamiltonian, and $\epsilon_{n}$ is the corresponding band energy. Using a local orbital basis set, an eigenstate can be expanded as

$$
\left|\psi_{n}\right\rangle=\sum_{i, \alpha} C_{i \alpha}^{(n)}|i \alpha\rangle
$$
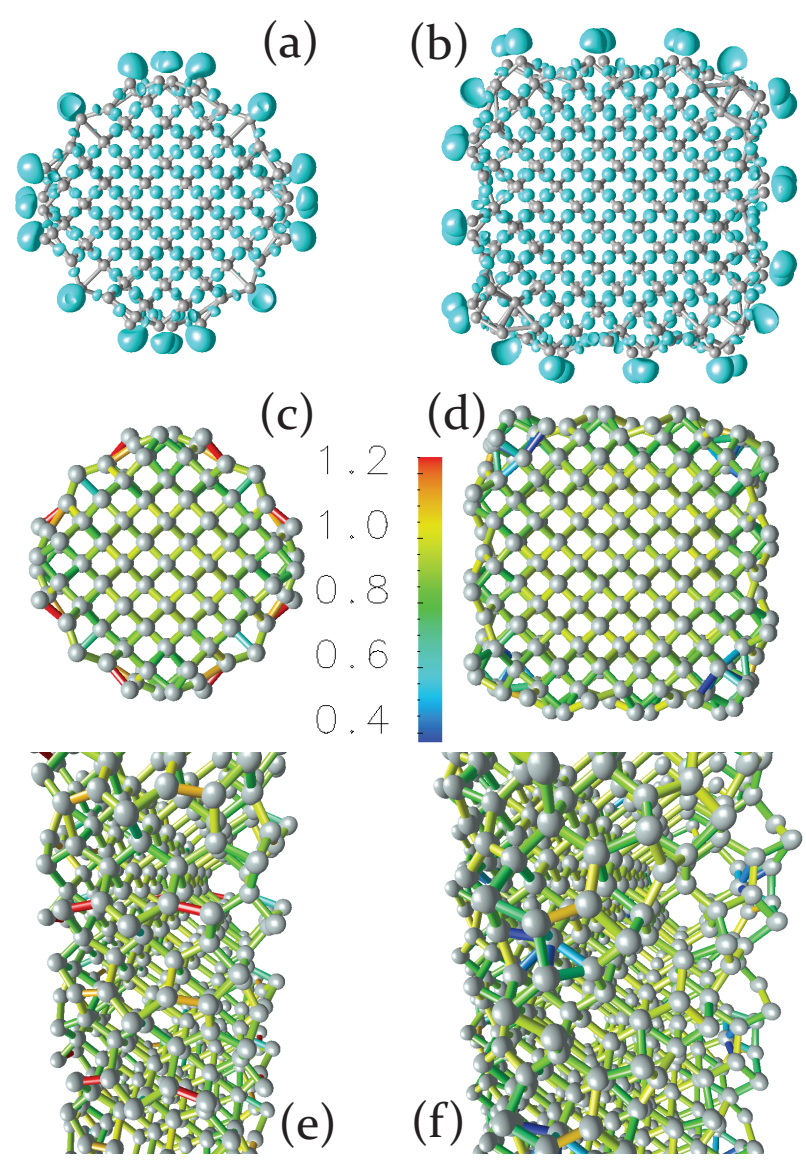

FIG. 2: (Color online) Electronic-structure calculations of $\mathrm{Si}<001>$ nanowires. (a)-(b) The electron localization function plots $(E L F=0.90$ isosurface) for the 1.45 and $1.87 \mathrm{~nm}$ wires respectively, (c)-(d) the cross sections, and (e)-(f) the side views for the same wires with the bond order (BO) in color. The BOs are normalized by the bulk value.

where $C_{i \alpha}^{(n)}$ is the contribution of orbital $\alpha$ at atomic site $i$ to the $n$-th band (eigenvectors). Then the bond order between atoms $i$ and $j$ can be defined $\operatorname{as}^{28}$

$$
\begin{gathered}
\Theta_{i j}=\sum_{\alpha, \beta} \theta_{i \alpha, j \beta} \theta_{j \beta, i \alpha}, \\
\theta_{i \alpha, j \beta}=\sum_{n} C_{i \alpha}^{(n)} C_{j \beta}^{(n) *} .
\end{gathered}
$$

Here in DFT, eigenvectors are replaced by the projection of wavefunctions (planewaves) onto local orbitals:

$$
C_{i \alpha}^{(n)}=\left\langle i \alpha \mid \psi_{n}\right\rangle \text {. }
$$

In applying this analysis to the Si nanowires, we find that many of the bonds to surface atoms have a bond order comparable to that of a bulk bond. Only the bonds in an environment far from the bulk diamond cubic structure show deviation from the bulk value. For example, the 1.45-nm wire has surface atoms with the coordination of 2 (bonds colored red in Fig. 2(c) and (e)). One of the bonds attached to these surface atoms shows a 


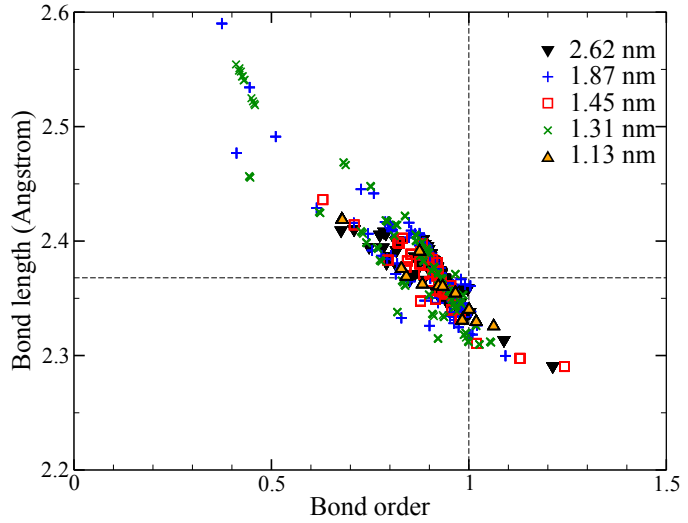

FIG. 3: (Color online) Scatter plot of bond order vs. bond length for the bare $\mathrm{Si}<001>$ nanowires. The dashed lines mark the bulk bond order and the bulk equilibrium bond length. Substantially low BOs are found for the 1.31 and $1.87-\mathrm{nm}$ wires, and high BOs for the 1.45 and 2.62-nm wires.

$20 \%$ higher BO than the bulk value but the other bond does not. The charge redistribution is apparent but not symmetric: bond order is not simply equivalent to coordination number. Another example is the bonds with a substantially lower BO in the 1.87-nm wire (bonds colored blue in Fig. 2(d) and (f)): these are some of the six bonds connected to the same over-coordinated atom. In addition, the backbonds under the surface experience a bond-order drop due to the surface relaxation in which the bonds typically lengthen. Such local-structure dependence may be seen in the correlation between bond order and length in Fig. 3.

This linear bond-order-bond-length relationship supports in part the conventional picture of the relationship between bond order, length and strength. ${ }^{12}$ The longer bonds have lower BO. It is unexpected, however, that the BO never significantly exceeds the bulk value, instead of approaching a value of 2 for undercoordinated atoms. Another unexpected aspect of Fig. 3 is the $\sim 10 \%$ deviation from the bulk bond order at equilibrium. This shift may be related to quantum confinement for two reasons. First, even the centermost atoms show a slight decrease in bond order. Second, it is known that the electronic structure of a nanostructure depends on thickness, ${ }^{29}$ and even neighboring interior atoms have a different local electronic structure. ${ }^{30}$ These differences in the local electronic structure of neighboring atoms affect bond order, as seen in Eq. (3). However, the bond order shift is not the primary source of softening. The average change is only $5 \sim 10 \%$ regardless of the wire size, whereas the softening is as large as $50 \%$ for the bare wires tested. The small bond-order shift means no substantial deviation from the bulk bond strength, and the size-dependence of the Young's modulus should not be any different for bare and $\mathrm{H}$-passivated wires, as evidenced in Fig. 1. Therefore, the softening is considered to be weak in that there is no other effects than the increased surface area to volume regardless of the surface condition, and it is propor-

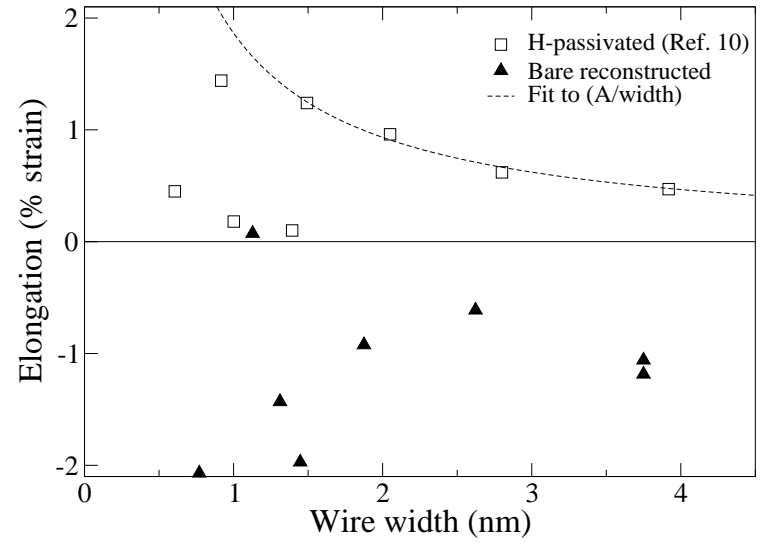

FIG. 4: Equilibrium elongation of the $\mathrm{Si}<001>$ nanowire as a percent of the wire length at the bulk lattice constant. The A/width curve is a fit to four data points from $1.49 \mathrm{~nm}$ and larger H-passivated wires.

tional to the surface-to-volume ratio. ${ }^{10}$ As the surface area to volume ratio increases, the population of interior atoms relative to surface atoms, and hence "load-bearing bonds", is decreased giving the solid curve in Fig. 1.

It is instructive to consider other properties of the wires as a whole beyond the Young's modulus. We have seen that the H-passivated nanowires are longer at equilibrium than would be predicted based on the bulk lattice constant. ${ }^{10}$ The elongation increases as the wire width is decreased due to compressive surface stress, following different curves depending on the surface facet indices. In contrast, the surface layer of bare wires experiences tensile stress leading to the negative axial strains at equilibrium shown in Fig. 4. The magnitude of the surface stress for the bare wires varies substantially depending on the structure of the relaxed, reconstructed surface, so that the elongation shown in Fig. 4 is not a simple function of the wire width. The repulsive $\mathrm{H}-\mathrm{H}$ interactions on the $\{100\}$ facets lead to the compressive stress of the $\mathrm{H}$ passivated surfaces $;^{10}$ the reconstructed dimer patterns on the $\{100\}$ facets account in part for the tensile stress of the bare surfaces, but the complexity in the surface stress is not entirely understood from the current data.

Regarding the relationship between the chemical bonding (covalent vs. metallic) and the Young's modulus, we do not observe any correlation between the two. The band gap of the hydrogen-passivated wires is dominated by wire size, ${ }^{30,31}$ but the band gap of the bare wires turns out to be highly dependent on the surface structure, i.e. the reconstructed patterns and edge shapes. ${ }^{5,20}$ In general, all the bare wires tested here have a lower band gap than the bulk limit, and any systematic size dependence is not observed. It is remarkable then that the Young's moduli fall into a single curve regardless of the existence or the magnitude of a band gap.

For the H-passivated nanowires it was possible to construct a predictive model of the nanowire mechanics based on bulk and slab calculations; ${ }^{10}$ we expect that 
a similar model would work for the bare wires, but the form would need to be extended to include, on top of the accurate surface states, quantum confinement effects of one-dimensional structures, ${ }^{31}$ and the effects of wire edge geometry ${ }^{5}$ and strain $^{32,33}$ on the electronic structure. At this point, slab ${ }^{34}$ and semi-empirical ${ }^{35}$ calculations are informative, but not sufficient to accurately predict the behavior of bare wires, so first-principles calculations with explicit nanowire geometry are necessary.

In conclusion, we have studied the effect of surface state on the mechanics of $\mathrm{Si}<001>$ nanowires. The surface stress is strongly affected by surface state: the eigenstrain (equilibrium elongation) asymptotically approaches zero for H-passivated nanowires, but for bare nanowires up to $4 \mathrm{~nm}$ the eigenstrain is scattered without such definitive trend. However, the softening of the nanowires varies systematically in both cases, and remarkably the Young's modulus is the same down to $0.6 \mathrm{~nm}$. This is striking not only because the Young's modulus of $\mathrm{Si}$ nanowires is insensitive to the surface states, but because it is contrary to the conventional be- lief that the sign of the surface moduli is determined by the sign of surface stress. Large differences in the bond order of bare and passivated wires were not observed, because the lone pair electrons remain along the dangling bonds; indeed, this is why those dangling bonds that remain after the surface reconstruction are still highly reactive. In both cases, the weak softening of the Young's modulus with decreasing size is not related to the bond order, which differs somewhat, but results from the diminished load-bearing capacity of the bonds near the surface. This geometrical effect gives a size-dependent softening that is invariant to changes in the surface state.

We are grateful to LC computing for allocations on Zeus. B. L. would like to thank P. Leu and K. Cho for the tight binding code. This work was performed under the auspices of the U.S. Dept. of Energy by Lawrence Livermore National Laboratory under Contract DE-AC5207NA27344. B. L.'s work was supported by Basic Science Research Program through the National Research Foundation of Korea (NRF) funded by the Ministry of Education, Science and Technology (2011-0027136).
* Electronic address: robert.rudd@llnl.gov

1 H. Ibach, J. Vac. Sci. Technol. A 12, 2240 (1994).

2 R. Berger, E. Delamarche, H. P. Lang, Ch. Gerber, J. K. Gimzewski, E. Meyer, and H.-J. Güntherodt, Science 276, 2021 (1997).

3 J. Fritz, M. K. Baller, H. P. Lang, H. Rothuizen, P. Vettiger, E. Meyer, H. -J. Güntherodt, Ch. Gerber, and J. K. Gimzewski, Science 288, 316 (2000).

4 S. Ismail-Beigi and T. Arias, Phys. Rev. B 57, 11923 (1998).

5 R. Rurali and N. Lorente, Phys. Rev. Lett. 94, 026805 (2005).

6 J. Q. Broughton, C. A. Meli, P. Vashishta, and R. K. Kalia, Phys. Rev. B 56, 611 (1997).

7 R. E. Rudd and J. Q. Broughton, J. Mod. Sim. Microsys. 1, 29 (1999).

8 S. Cuenot, C. Frétigny, S. Demoustier-Champagne, and B. Nysten, Phys. Rev. B 69, 165410 (2004).

9 R. E. Miller and V. B. Shenoy, Nanotechnology 11, 139 (2000).

10 B. Lee and R. E. Rudd, Phys. Rev. B 75, 041305(R) (2007); 75, 195328 (2007).

11 L. G. Zhou and H. Huang, Appl. Phys. Lett. 84, 1940 (2004); L. Zhang and H. Huang, ibid. 89, 183111 (2006).

12 C. Q. Sun, B. K. Tay, X. T. Zeng, S. Li, T. P. Chen, J. Zhou, H. L. Bai, and E. Y. J. Jiang, J. Phys.: Condens. Matter 14, 7781 (2002).

13 R. E. Rudd, unpublished.

14 R. E. Rudd, Intl. J. on Multiscale Comput. Engin. 2, 203 (2004).

15 A. N. Cleland and M. L. Roukes, Appl. Phys. Lett. 69, 2653 (1996).

16 D. J. Chadi, Phys. Rev. Lett. 43, 43 (1979).

17 J. Ihm, D. H. Lee, J. D. Joannopoulos, and J. J. Xiong, Phys. Rev. Lett. 51, 1872 (1983).

18 N. Roberts and R. J. Needs, Surf. Sci. 236, 112 (1990).

19 W. E. Packard and J. D. Dow, Phys. Rev. B 55, 15643
(1997).

20 A. A. Stekolnikov, J. Furthmüller, and F. Bechstedt, Phys. Rev. B 65, 115318 (2002); 70, 045305 (2004).

21 The energy cutoff is $400 \mathrm{eV}$, and $8 \times 8$ and $13 \times 9$ uniform $k$-point meshes are used for the $\{100\}$ and $\{110\}$ surfaces respectively. For the bare wires, three $k$-points in the onedimensional irreducible Brillouin zone are used. The supercell has two cubic unit cells in the wire direction to accommodate the prescribed reconstruction patterns. The largest wire has 810 atoms fully relaxed.

${ }^{22}$ G. Kresse and J. Furthmüller, Phys. Rev. B 54, 11169 (1996).

23 P. E. Blöchl, Phys. Rev. B 50, 17953 (1994).

24 G. Kresse and D. Joubert, Phys. Rev. B 59, 1758 (1999).

25 J. P. Perdew, K. Burke, and M. Ernzerhof, Phys. Rev. Lett. 77, 3865 (1996)

26 A. D. Becke and K. E. Edgecombe, J. Chem. Phys. 92, 5397 (1990).

27 B. Silvi and A. Savin, Nature 371, 683 (1994).

28 M. Giambiagi, M. Giambiagi, D. R. Grempel, and C. D. Heynmann, J. Chim. Phys. 72, 15 (1975); I. Mayer, J. Mol. Struct.: THEOCHEM 149, 81 (1987).

29 S. C. Hong, J. I. Lee, and R. Wu, Phys. Rev. B 75, 172402 (2007).

30 T. Vo, A. J. Williamson, and G. Galli, Phys. Rev. B 74, 045116 (2006)

31 F. Buda, J. Kohanoff, and M. Parrinello, Phys. Rev. Lett. 69, 1272 (1992).

32 G. Peng and Y. Feng, Appl. Phys. Lett. 91, 083116 (2007).

33 P. W. Leu, A. Svizhenko, and K. Cho, Phys. Rev. B 77, 235305 (2008).

34 Y. Umeno, A. Kushima, T. Kitamura, P. Gumbsch, and J. Li, Phys. Rev. B 72, 165431 (2005).

35 D. E. Segall, S. Ismail-Beigi, and T. A. Arias, Phys. Rev. B 65, 214109 (2002). 